Radiologe 2010 · 50:589-590

DOI 10.1007/s00117-010-2034-5

Online publiziert: 24. Juni 2010

๑) Springer-Verlag 2010

\author{
W. Reith \\ Klinik für Diagnostische und Interventionelle Neuroradiologie, \\ Universitätsklinikum des Saarlandes, Homburg/Saar
}

\title{
Diagnostik und Therapie der Karotisstenose
}

Sehr geehrte Leserinnen und Leser,

die Inzidenz des Schlaganfalls beträgt ca. 165.00o pro Jahr für die gesamte Bundesrepublik Deutschland, $18 \%$ sind durch überwiegend arteriosklerotische Veränderungen an den extrakraniellen hirnversorgenden Arterien, v. a. an der Karotisbifurkation, bedingt.

Zur Beurteilung des Stenosegrads und deren Ursachen sind zuverlässige bildgebende Verfahren notwendig. Das Spektrum an Pathologien reicht von Arteriosklerose, Dissektion, Vaskulitiden, fibromuskulärer Dysplasie bis hin zu Tumoren.

Neben der Duplexsonographie haben Schnittbildverfahren wie die CT-Angiographie und MR-Angiographie zunehmend an Bedeutung gewonnen und sind hinsichtlich ihrer diagnostischen Qualität der digitalen Subtraktionsangiographie fast gleichwertig.

Die erfolgreiche Behandlung eines Patienten mit symptomatischer Karotisstenose mittels chirurgischer Thrombendarteriektomie wurde 1954 erstmals publiziert. Durch randomisierte multizentrische Studien konnte zunächst für symptomatische, später aber auch für asymptomatische Patienten die Überlegenheit der chirurgischen gegenüber einer allein konservativen Therapie nachgewiesen werden. Die perkutane Angioplastie mit Stentimplantation hat sich seit ihrer Erstbeschreibung durch Mathias als wenig invasives Verfahren mittlerweile als alternative Behandlungsmethode etabliert.

Das Hauptrisiko bei beiden Behandlungsarten ist das Auftreten eines Schlaganfalls. Eine erst kürzlich veröffentlichte
Studie (Carotid Revascularization Endarterectomy versus Stenting Trial - CREST) wurde an 117 Zentren in Nordamerika durchgeführt und schloss 2502 Patienten mit $>70 \%$ iger Stenose ein.

Etwa die Hälfte der Patienten hatte bereits einen Schlaganfall erlitten. Die europäische International Carotid Stenting Study (ICSS) umfasste 1713 symptomatische Patienten mit hohem Anteil an $>70 \%$ igen Stenosen. Die Ergebnisse zeigten, dass es in den ersten 30 Tagen nach der Stentimplantation zu einer erhöhten Rate periprozeduraler Schlaganfälle kam (4,1 vs. $2,3 \%$ in der CREST-Studie und 9,1 vs. $7,7 \%$ in der ICSS-Studie. Die meisten dieser Schlaganfälle waren nicht beeinträchtigend. In beiden Studien zeigte sich jedoch ein gehäuftes Auftreten von Myokardinfarkten (2,3 vs. $1,1 \%$ in der CREST-Studie; 0,5 vs. $0 \%$ in der ICSS-Studie).

Die Schlussfolgerung der US-Autoren war, das der Stent eine sichere und alternative Methode zur Operation darstellt, v. a. für Patienten $<70$ Jahre, bei denen die Ergebnisse der Stentimplantation besser waren.

Auch im Langzeitverlauf findet sich kein Unterschied hinsichtlich der Freiheit von ipsilateralen Schlaganfällen. Zusätzlich liegen mehrere Publikationen großer multizentrischer Register vor. In 2 prospektiv geführten Registern, die beide den Hospitalzeitraum erfassen, zeigte sich eine Abnahme der kombinierten Häufigkeit von Tod und Schlaganfall im Zeitraum Juli 1999 bis September 2000 gegenüber dem Zeitraum Oktober 2000 bis Juni 2003 (4,6 vs. 2,1\%). Dies zeigt, dass mit zunehmender Erfahrung der Untersucher und besseren Techniken, wie z. B. Protek- 
tionssystemen mit verbessertem Stentdesign und Einführungssystemen, eine relativ niedrige Komplikationsrate erreicht werden kann. Die früher angeführten Instent-Restenosen konnten in mehreren Publikationen unabhängig vom verwendeten Stent mit 3 bis $<7 \%$ dokumentiert werden. Jenseits des 30-Tages-Zeitraums seit dem Eingriff entspricht die Rate ipsilateraler neurologischer Defizite derjenigen nach Karotisthrombendarteriektomie.

Zielgruppe für die interventionelle Karotisangioplastie ist im Wesentlichen die gleiche Patientenklientel, die für eine Karotisthrombendarteriektomie in Frage kommt. In 2 randomisierten prospektiven Studien hat sich jedoch gezeigt, dass jüngere Patienten mit <68 Jahren eher von der stentgestützten Karotisangioplastie profitieren.

Wegen der dokumentierten inversen Beziehung zwischen Interventionszahl und Komplikationsrate sind an die interventionell tätigen Ärzte hohe Qualitätsansprüche zu stellen. Die von der American Heart Association (AHA) für die Karotischirurgie geforderten Komplikationsmaxima sollten auch von den Interventionalisten keinesfalls überschritten werden. Das Erreichen einer periinterventionellen Komplikationsrate von $<3 \%$ für asymptomatische bzw. $<6 \%$ für symptomatische Patienten sollte unbedingt von externer unabhängiger Seite kontrolliert werden.

Voraussetzung hierfür ist eine allgemeine große Interventionserfahrung bei arteriosklerotischen Gefäßerkrankungen. Unterstützt kann dies durch strukturierte Trainingsprogramme werden. Die interdisziplinäre Zusammenarbeit bei der Indikationsstellung ist von herausragender Bedeutung. Alle Patienten sollten vor und nach dem Eingriff von einem Neurologen untersucht werden.

Die Debatte um die Behandlung der Karotisstenose ist immer noch kontrovers. Bei richtiger Indikationsstellung ist der Nutzen für den Patienten jedoch evident. In den Händen eines erfahrenen Interventionalisten und/oder Chirurgen stellt die Behandlung einer Karotisstenose ein relativ sicheres Verfahren dar. Wichtig ist jedoch, dass der Interventionalist und auch der Chirurg auf dem Gebiet der Karotis- stenosetherapie erfahren sind. Für den Interventionalisten hat sich gezeigt, dass eine deutliche Lernkurve existiert und erst ab ca. 8o Eingriffen an der Halsschlagader eine genügende Sicherheit vorhanden ist, um komplikationsarm zu arbeiten.

Ihr

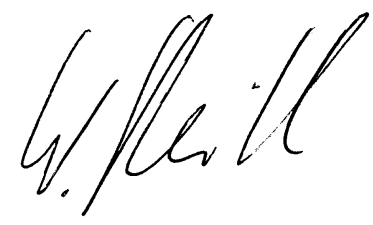

Prof. Dr. Wolfgang Reith 